\title{
Alternative Fistula Risk Score and First Postoperative Day Drain Fluid Amylase as Predictors of Pancreatic Fistula After Pancreatoduodenectomy
}

Karina Scalabrin Longo ( $\nabla$ karina.longo@yahoo.com.br)

Faculdade de Medicina do ABC

Thiago Bassaneze

Faculdade de Medicina do ABC

Rogério Tadeu Palma

Faculdade de Medicina do ABC Jaques Waisberg

Faculdade de Medicina do ABC

\section{Research Article}

Keywords: Pancreatic fistula, Pancreatoduodenectomy, Postoperative complications, Prognosis

Posted Date: July 27th, 2021

DOl: https://doi.org/10.21203/rs.3.rs-726054/v1

License: (c) (i) This work is licensed under a Creative Commons Attribution 4.0 International License.

Read Full License 


\section{Alternative fistula risk score and first postoperative day drain fluid amylase as predictors of pancreatic fistula after pancreatoduodenectomy}

Karina Scalabrin Longo ${ }^{1 *}$, Thiago Bassaneze ${ }^{2+}$, Rogério Tadeu Palma ${ }^{3+}$, Jaques Waisberg $^{4+}$

1,2,3,4 Department of Gastrointestinal Surgery, Faculdade de Medicina do ABC, Santo André, São Paulo, Brazil

${ }^{+}$these authors contributed equally to this work

*Corresponding author: Karina Scalabrin Longo

Postal address: Rua Professor Alexandre Correia, 370, ap 41, Jardim Vitória Régia, São Paulo, SP,

Zipcode 05657-230, Brazil

Phone number: +55 (11) 960597807

Fax number: +55 (11) 37587430

E-mail: karina.longo@yahoo.com.br

Running head: Predictors for postoperative pancreatic fistula 


\begin{abstract}
Objectives: The alternative fistula risk score (aFRS) and the first postoperative day drain fluid amylase (DFA) are predictors of the occurrence of clinically relevant postoperative pancreatic fistula (CR-POPF). No consensus has been reached on which of the scores is a better predictor; moreover, their combined predictive power remains unclear. To our knowledge, this association had not yet been studied.

Methods: This study assessed the predictive effect of aFRS and/or DFA on CR-POPF in a retrospective cohort of 58 patients following PD. The Shapiro-Wilk and the Mann-Whitney tests were applied for assessing the distribution of the samples and for comparing the medians, respectively. The receiver operating characteristics (ROC) curve and the confusion matrix were used to analyze the predictive models.

Results: The aFRS values were not statistically different between patients in the CR-POPF and non-CR-POPF groups (Mann-Whitney U test: 59.5, p=0.12). The DFA values were statistically different between the CRPOPF and non-CR-POPF groups (Mann-Whitney U test: 27, p=0.004). The aFRS and DFA were independently less predictive for CR-POPF, compared to combined aFRS + DFA.

Conclusions: The combined model involving aFRS $>20 \%+\mathrm{DFA} \geq 5,000 \mathrm{U} / \mathrm{L}$ was the most effective predictor of CR-POPF occurrence following PD.
\end{abstract}

\title{
Keywords
}

Pancreatic fistula; Pancreatoduodenectomy; Postoperative complications; Prognosis 


\section{Introduction}

The high morbidity and mortality rates of pancreatoduodenectomy (PD), performed for the treatment of periampullary diseases, are mainly associated with pancreaticojejunal anastomosis. Of all anastomoses performed during PD, pancreaticojejunal anastomosis is the most fragile and is susceptible to complications such as clinically relevant postoperative pancreatic fistula (CR-POPF). CR-POPF occurs in 3-45\% of PD procedures and increases hospital stay, readmissions, and reoperations. The occurrence of CR-POPF can also delay the start of adjuvant therapy and reduce the overall survival of patients following PD for malignant diseases. Moreover, it consistently increases health care $\operatorname{costs}^{1-4}$. The critical factor for predicting the occurrence of CR-POPF is risk stratification by assessing patient- and procedure-related criteria and creating a fistula risk profile $^{3}$.

The first internationally accepted definition of postoperative pancreatic fistula (POPF) was established in 2005 by the International Study Group of Pancreatic Fistula (ISGPF). ${ }^{1}$ It was defined as abdominal drain output of any measurable volume of fluid on and after postoperative day 3 with amylase levels in the drained fluid 3 times higher than the institutional normal serum amylase upper limit. This classification stratified patients into grade A (benign clinical course), grade B (patients at moderate risk), and grade C (critical patients requiring invasive intervention). ${ }^{1} \mathrm{~A} 2016$ review of the definition of $\mathrm{POPF}^{2}$ considered grades $\mathrm{B}$ and $\mathrm{C}$ as $\mathrm{CR}$ POPF and renamed grade A as biochemical leak (BL), a pancreatic secretion extravasation without clinical implications. The grade B POPF is the fistula itself, and requires changes in postoperative management, including oral fasting, enteral or parenteral diet support, and antibiotic therapy in cases of infection. Moreover, it may also require an invasive intervention with percutaneous or endoscopic drainage of intra-abdominal collections. Grade C POPF occurs when grade B POPF is complicated with organ failure, clinical instability, need for reoperation, or death. ${ }^{2}$ These terms are summarized in Table 1.

The alternative fistula risk score (aFRS) was described in $2017^{4}$ and externally validated in multicenter studies. $^{4-9}$ This score considers three predictive factors of CR-POPF: pancreatic parenchyma texture, diameter of the main pancreatic duct (Wirsung's duct), and the patient's body mass index (BMI). The aFRS classifies the risk for developing CR-POPF into three groups: low- (aFRS 0\% to 5\%), intermediate- (aFRS >5\% to 20\%), and high-risk (aFRS > 20\%) groups. ${ }^{4}$ These terms are summarized in Table 1. CR-POPF occurrence can also be predicted by measuring the first postoperative day drain fluid amylase (DFA). ${ }^{10,11}$ Visual changes in the appearance of the drain fluid only become evident from postoperative day $5,{ }^{12}$ whereas amylase levels in the drain fluid increase already on postoperative day 1 due to its early and imperceptible extravasation through the 
anastomosis. Several cutoff values of DFA, ${ }^{11-24}$ ranging from $90 \mathrm{U} / \mathrm{L}^{21}$ to $5,000 \mathrm{U} / \mathrm{L},{ }^{12}$ have been described for determining the high risk of developing CR-POPF. The most cited cutoff is $\geq 5,000 \mathrm{U} / \mathrm{L} ;{ }^{12}$ thus, motivating surgeons to remove the abdominal drain early when DFA values are below the aforementioned cutoff. ${ }^{25}$

Several studies ${ }^{4,10-12}$ have pointed out the importance of measuring the aFRS and DFA. With varying diagnostic values, ${ }^{4,11-24}$ the determination of these scores facilitates a more individualized management, considering if the patient is a low- or high-risk of developing CR-POPF. ${ }^{3,4,25-29}$ However, there is still no consensus on which of these scores is better, and whether combining both scores would result in increased accuracy in the selection of patients with a high risk of developing CR-POPF. To our knowledge, this association had not yet been studied.

Table 1. Postoperative pancreatic fistula with its definition and grades proposed by the International Study Group of Pancreatic Surgery ${ }^{2}$; and the alternative fistula risk score with its parameters, calculator and risk groups. ${ }^{4}$

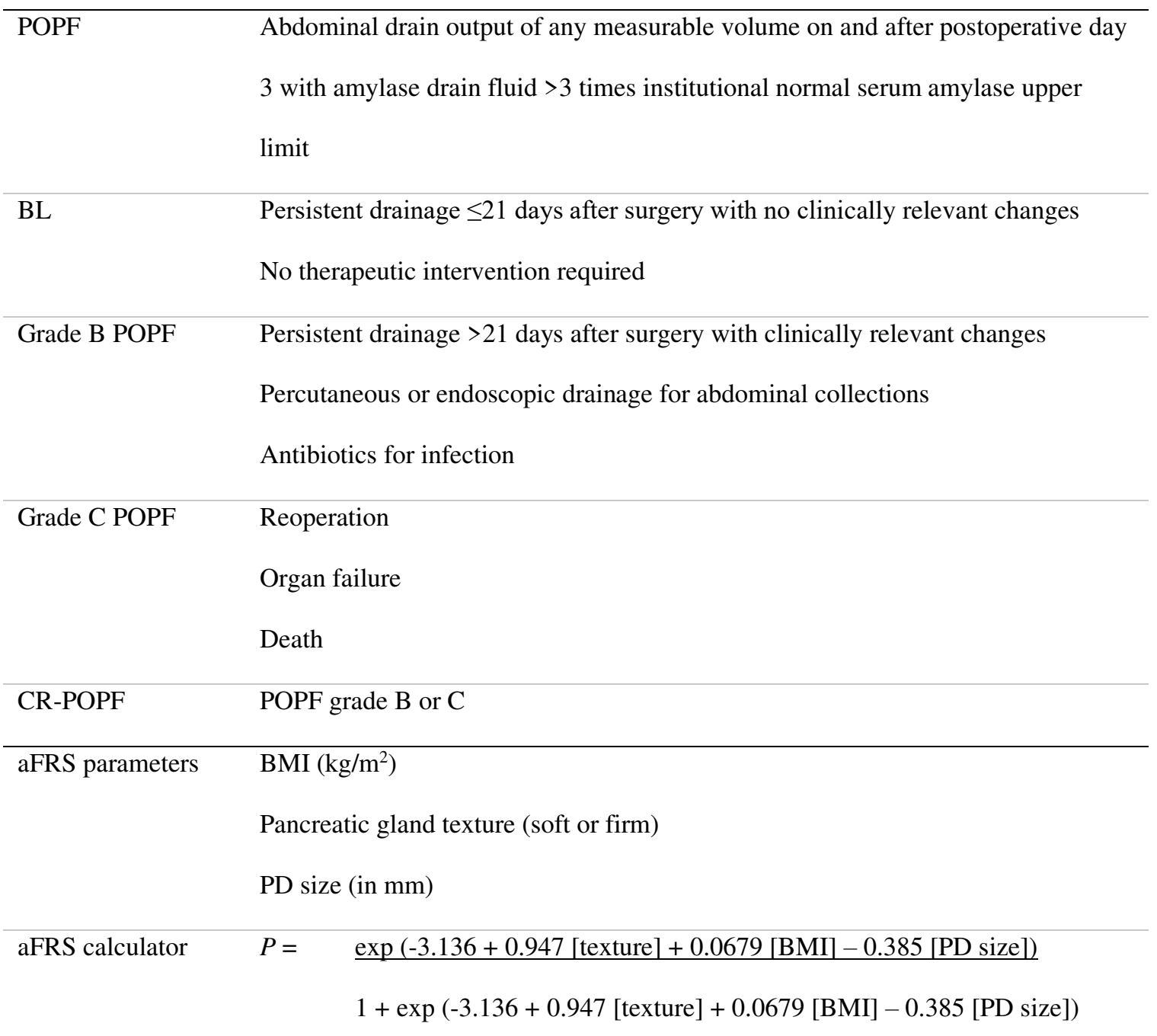


texture $1=$ soft and $0=$ firm, PD size in mm (truncated at 5)

aFRS risk groups $\quad$ Low risk of CR-POPF (aFRS $0 \%$ to $5 \%$ )

Intermediate risk of CR-POPF (aFRS $>5 \%$ to $20 \%$ )

High risk of CR-POPF (aFRS $\geq 20 \%$ )

POPF: postoperative pancreatic fistula; BL: biochemical leak; CR-POPF: clinically relevant postoperative pancreatic fistula; aFRS: alternative fistula risk score; BMI: body mass index; PD: pancreatic duct; P: probability; exp: exponential function.

\section{Materials and Methods}

\section{Study design, patients, and data collection}

We carried out a retrospective cohort of 58 patients who underwent PD for malignant or benign periampullary disorders. The surgeries were performed between January 2016 and October 2019 at the Department of Gastrointestinal Surgery of the ABC Medical School in São Paulo, Brazil. The study was submitted to and approved by the institution's Research Ethics Committee, on a countrywide platform (Comitê de Ética e Pesquisa da Faculdade de Medicina do ABC, Plataforma Brasil - number 28434719.6.0000.0082) and was conducted in accordance with the Declaration of Helsinki. The patients' medical records were analyzed through the institution's database, and the collected information was anonymized. A waiver for obtaining informed consent was acquired and included in the approval process by the same national platform (Comitê de Ética e Pesquisa da Faculdade de Medicina do ABC, Plataforma Brasil).

The collected biodemographic data included the following information: age, gender, ethnicity, body mass index (BMI), smoking history, alcoholism, and associated comorbidities (systemic arterial hypertension [SAH], diabetes mellitus [DM], vasculopathies, and heart and lung diseases). The preoperative data also considered individual weight loss, neoadjuvant treatment, presence of obstructive jaundice, and the presence of biliary prostheses.

Intraoperative events were assessed considering the surgical time, type of pancreaticojejunal anastomosis, use of transanastomotic stents, multivisceral resection, vascular reconstruction, use of abdominal drains, and transfusion of hemoconcentrated blood. The surgeon intraoperatively determined the diameter of the Wirsung's duct and the pancreatic parenchyma texture by palpation of the pancreatic gland..$^{30,31}$

The postoperative progression was based on the length of hospital stay, duration of abdominal drain use, use of antibiotics and parenteral nutrition, and occurrence of pancreatic, biliary, or lymphatic fistulas. The 
clinical or surgical complications were assessed individually based on the need for interventions, such as percutaneous drainage of intra-abdominal collections or reoperation.

The stratification according to POPF grades followed the ISGPF guidelines. ${ }^{2}$ The CR-POPF risk assessment used two predictive scores that had been previously described in the literature: aFRS ${ }^{4}$ and DFA. ${ }^{10,11}$ The aFRS was calculated using the online calculator available at www.pancreascalculator.com. ${ }^{4}$ The abdominal drain fluid was collected on postoperative day 1 to measure the amylase levels. ${ }^{10,11}$ The original paper that describes aFRS ${ }^{4}$ classified patients with aFRS> $20 \%$ as having a high risk for CR-POPF; we adopted this value in the present study as well. A DFA of $\geq 5,000 \mathrm{U} / \mathrm{L}$ is considered to indicate a high risk for CR-POPF ${ }^{12}$; we also adopted this value in the present study.

The study included adult patients of both genders who underwent elective PD due to malignant or benign periampullary disorders. We excluded patients with medical records having insufficient information for calculating the aFRS or without a DFA value. The medical records of 58 patients were initially reviewed and 18 were excluded from the study for insufficient information (Fig. 1).

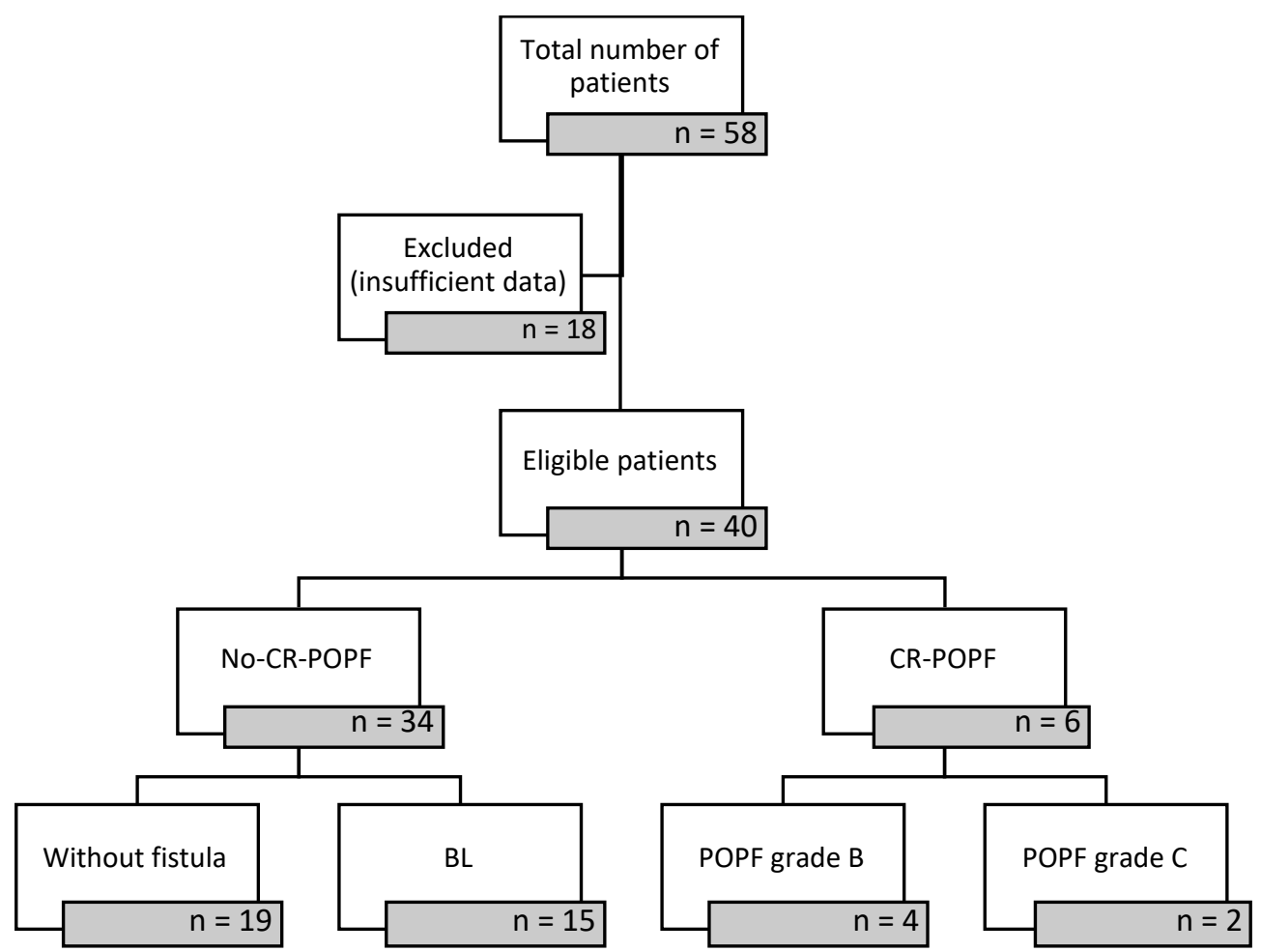

Fig. 1. Selection of the studied patients and occurrence or not of clinically relevant postoperative pancreatic fistula. 
CR-POPF: clinically relevant postoperative pancreatic fistula; BL: biochemical leak; POPF: postoperative pancreatic fistula, Grade B POPF: fistula requiring a change in postoperative management. Grade C POPF: grade B with organ failure, clinical instability, need for reoperation, or death.

\section{Statistical analysis}

This study assessed the correlation between aFRS and/or DFA and the occurrence of CR-POPF by performing a descriptive analysis of the data comparing the scores individually (aFRS or DFA) or combined (aFRS + DFA). The means, medians, and percentage distribution of each variable were calculated, and the corresponding boxplots were traced. The Shapiro-Wilk normality test was applied to assess the distribution of the samples and the two-tailed non-parametric Mann-Whitney test to compare the medians. The receiver operating characteristic (ROC) curve and the confusion matrix were used to analyze the predictive models. Performance was determined by the metrics sensitivity (Se), specificity (Sp), positive predictive value (PPV), negative predictive value (NPV) and accuracy, and area under the curve (AUC). RStudio version 1.2.533 (RStudio, Boston, MA, USA) and Jupyterlab version 1.1.4 (Anaconda, Austin, TX, USA) were used for data analysis.

\section{Results}

\section{Biodemographic data}

The patients were divided into two groups: those who evolved with (CR-POPF group) and without (non-CR-POPF group) CR-POPF. The biodemographic, surgical, and pathological data are summarized in Table 2.

All surgeries were PDs (Whipple procedure) and had a curative intent in cases of malignant neoplasia. Laparotomy was the access route used in all patients. The reconstruction strategy consisted of a single loop with a duct-to-mucosa pancreaticojejunal anastomosis. Abdominal drainage was performed with a silicone tubulolaminar drain placed near the pancreaticojejunal anastomosis. Multivisceral and vascular resection was performed in $1(2.5 \%)$ case. The mean surgical time was $338.5 \pm 123.42 \mathrm{~min}$ (150 to $630 \mathrm{~min})$. The mean estimated intraoperative blood loss was $570 \pm 427.71 \mathrm{~mL}$ (50 to $1500 \mathrm{~mL}$ ). The Wirsung's duct diameters were $\leq 3 \mathrm{~mm}$ in $26(65 \%)$ patients and $>3 \mathrm{~mm}$ in $14(35 \%)$. The pancreatic parenchyma texture was considered firm in $18(45 \%)$ patients and softened in $22(55 \%)$. 
Postoperatively, biliary fistula was observed in 3 (7.5\%) cases; 2 were surgically and 1 was conservatively treated, and fistulas were resolved in all cases. Lymphatic fistula was observed in 2 (5\%) cases, which both progressed favorably after clinical treatment.

There were $6(15 \%)$ cases of pancreatic fistula, namely 4 (10\%) with grade B and $2(5 \%)$ with grade C. CR-POPF was not observed in $34(85 \%)$ cases, $15(37.5 \%)$ had BL and $19(47.5 \%)$ did not have any type of fistula. The 2 patients with grade C POPF died from multiple organ failure, 1 on postoperative day 6 due to acute renal failure and systemic inflammatory response syndrome (SIRS), and the other on postoperative day 25 after reoperation. Of the 4 patients with grade B POPF, 3 underwent percutaneous drainage of abdominal collections and 1 followed conservative clinical treatment, all of them with good progression.

The mean length of hospital stay was 16 and 12.9 days in the CR-POPF and non-CR-POPF groups, respectively (Mann-Whitney test; $\mathrm{p}>0.05$ ). The mean duration of abdominal drain use was 15.6 and 11.9 days in the CR-POPF and non-CR-POPF groups, respectively (Mann-Whitney test; $\mathrm{p}>0.05$ ).

Table 2. Clinical characteristics and pre-, intra- and postoperative aspects of patients after duodenopancreatectomy (DP) due to periampullary disorders.

\begin{tabular}{|c|c|c|c|}
\hline Biodemographic variables & $\begin{array}{l}\text { non-CR-POPF } \\
(\mathrm{N}=34)\end{array}$ & $\begin{array}{l}\text { CR-POPF } \\
(\mathrm{N}=6)\end{array}$ & $\begin{array}{l}\text { Total } \\
(\mathrm{N}=40)\end{array}$ \\
\hline \multicolumn{4}{|l|}{ Preoperative } \\
\hline Female & $18(52.9 \%)$ & $5(83.3 \%)$ & $23(57.5 \%)$ \\
\hline Male & $16(47.1 \%)$ & $1(16.7 \%)$ & $17(42.5 \%)$ \\
\hline Age (years) & $59.4(22-76)$ & $58(34-69)$ & $59.2(22-76)$ \\
\hline BMI $\left(\mathrm{kg} / \mathrm{m}^{2}\right)$ & $24.8(17-35)$ & $27.2(21-35)$ & $25.2(17-35)$ \\
\hline \multicolumn{4}{|l|}{ Ethnicity } \\
\hline White & $22(64.7 \%)$ & $4(66.7 \%)$ & $26(65 \%)$ \\
\hline Brown & $10(29.4 \%)$ & $1(16.7 \%)$ & $11(27.5 \%)$ \\
\hline Black & $2(5.9 \%)$ & $1(16.7 \%)$ & $3(7.5 \%)$ \\
\hline Smoking & $9(26.5 \%)$ & $2(33.3 \%)$ & $11(27.5 \%)$ \\
\hline Alcoholism & $4(11.7 \%)$ & $0(0 \%)$ & $4(10 \%)$ \\
\hline Associated comorbidities & $19(55.9 \%)$ & $4(66.7 \%)$ & $23(57.5 \%)$ \\
\hline Weight loss $>10 \%$ & $18(52.9 \%)$ & $2(33.3 \%)$ & $20(50 \%)$ \\
\hline
\end{tabular}




\section{Intraoperative}

Pancreatic parenchyma texture

\begin{tabular}{lccc}
\hline Firm & $15(44.1 \%)$ & $3(50 \%)$ & $18(45 \%)$ \\
\hline Softened & $19(55.9 \%)$ & $3(50 \%)$ & $22(55 \%)$ \\
\hline Pancreatic duct diameter & & & \\
\hline$\leq 3 \mathrm{~mm}$ & $20(58.8 \%)$ & $6(100 \%)$ & $26(65 \%)$ \\
$>3 \mathrm{~mm}$ & $14(41.2 \%)$ & $0(0 \%)$ & $14(35 \%)$
\end{tabular}

\section{Postoperative}

\begin{tabular}{|c|c|c|c|}
\hline \multicolumn{4}{|l|}{ Histopathology } \\
\hline Pancreatic head carcinoma & $16(47.1 \%)$ & $2(33.3 \%)$ & $18(45 \%)$ \\
\hline Duodenal papilla carcinoma & $10(29.5 \%)$ & $3(50 \%)$ & $13(32.5 \%)$ \\
\hline Chronic pseudotumoral pancreatitis & $3(8.8 \%)$ & $0(0 \%)$ & $3(7.5 \%)$ \\
\hline Duodenal cancer & $2(5.9 \%)$ & $1(16.7 \%)$ & $3(7.5 \%)$ \\
\hline Distal cholangiocarcinoma & $1(2.9 \%)$ & $0(0 \%)$ & $1(2.5 \%)$ \\
\hline Frantz tumor & $1(2.9 \%)$ & $0(0 \%)$ & $1(2.5 \%)$ \\
\hline IPMN & $1(2.9 \%)$ & $0(0 \%)$ & $1(2.5 \%)$ \\
\hline \multicolumn{4}{|l|}{ Pancreatic fistula } \\
\hline CR-POPF & & & $6(15 \%)$ \\
\hline Grade B POPF & $0(0 \%)$ & $4(66.7 \%)$ & $4(10 \%)$ \\
\hline Grade C POPF & $0(0 \%)$ & $2(33.3 \%)$ & $2(5 \%)$ \\
\hline non-CR-POPF & & & $34(85 \%)$ \\
\hline $\mathrm{BL}$ & $15(44.1 \%)$ & $0(0 \%)$ & $15(37.5 \%)$ \\
\hline No fistula & $19(55.9 \%)$ & $0(0 \%)$ & $19(47.5 \%)$ \\
\hline Biliary fistula & $3(8.8 \%)$ & $0(0 \%)$ & $3(7.5 \%)$ \\
\hline Lymphatic fistula & $2(5.9 \%)$ & $0(0 \%)$ & $2(5 \%)$ \\
\hline Length of hospital stay (days) & 12.9 & 16 & 13.4 \\
\hline Duration of drain use (days) & 11.9 & 15.6 & 12.5 \\
\hline Percutaneous drainage & $0(0 \%)$ & $3(50 \%)$ & $3(7.5 \%)$ \\
\hline
\end{tabular}




\begin{tabular}{llcl}
\hline Reoperation & $2(5.9 \%)$ & $1(16.7 \%)$ & $3(7.5 \%)$ \\
\hline Death & $0(0 \%)$ & $2(33.3 \%)$ & $2(5 \%)$
\end{tabular}

CR-POPF: clinically relevant postoperative pancreatic fistula; BMI: body mass index; IPMN: intraductal papillary mucinous neoplasia; BL: biochemical leak; POPF: postoperative pancreatic fistula; Grade B POPF: fistula that requires changes in postoperative management; Grade C POPF: grade B with organ failure, clinical instability, need for reoperation, or death.

Note: Due to the small number of patients, $p$ values were not shown in Table 3. They were calculated by Fisher's exact test due to the low volume of samples. The literature recommends that statistical treatment should be applied to at least 5 cases, and it is noted that for some parameters, the fistula group has only 1 or 2 patients, obtaining an unreliable $\mathrm{p}$ value that can lead to confusion in interpretation of the data.

\section{Correlation between aFRS and CR-POPF}

The mean aFRS was $22.38 \%$ and $12.23 \%$ in the CR-POPF and non-CR-POPF groups, respectively. We classified $6(15 \%)$ patients as low (aFRS 0\% to 5\%), $25(62.5 \%)$ as intermediate (aFRS >5 to 20\%), and 9 $(22.5 \%)$ as high risk (aFRS $>20 \%$ ) for CR-POPF. Considering aFRS for risk stratification of the sample population, none of the patients at low, $2(8 \%)$ of the intermediate, and 4 (44.4\%) of the high risk developed CR-POPF (Fig. 2). 
30

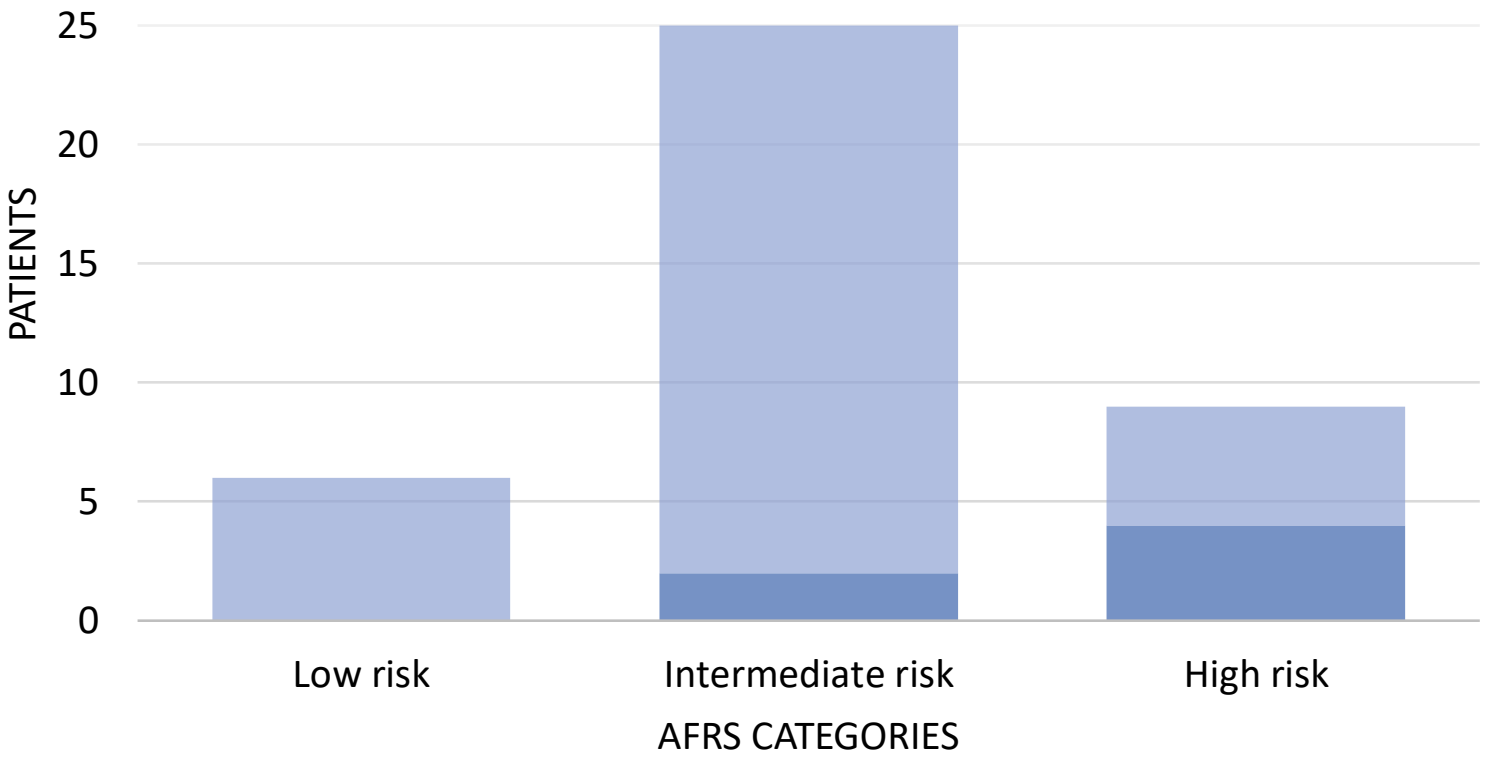

Fig. 2. Analysis of the distribution of aFRS categories in relation to the occurrence of CR-POPF. The patients were divided into three columns, according to aFRS categories: those with low- (aFRS 0\% to 5\%), those with intermediate- (aFRS $>5 \%$ to $20 \%$ ), and those with high-risk (aFRS $>20 \%$ ) for developing CR-POPF. Each column was divided into patients who developed CR-POPF (dark blue) and patients who not developed CR-POPF (light blue).

aFRS: alternative fistula risk score; CR-POPF: clinically relevant postoperative pancreatic fistula.

The analysis of the variability of aFRS in relation to the presence of CR-POPF demonstrated that the median aFRS was higher in the CR-POPF group compared to that in the non-CR-POPF group (Fig. 3).

The distribution of the aFRS values was non-parametric ( $\mathrm{p}<0.05$; Shapiro-Wilk normality test). There were no significant differences between the CR-POPF and non-CR-POPF groups (Mann-Whitney U test: 59.5, $\mathrm{p}=0.12)$. 


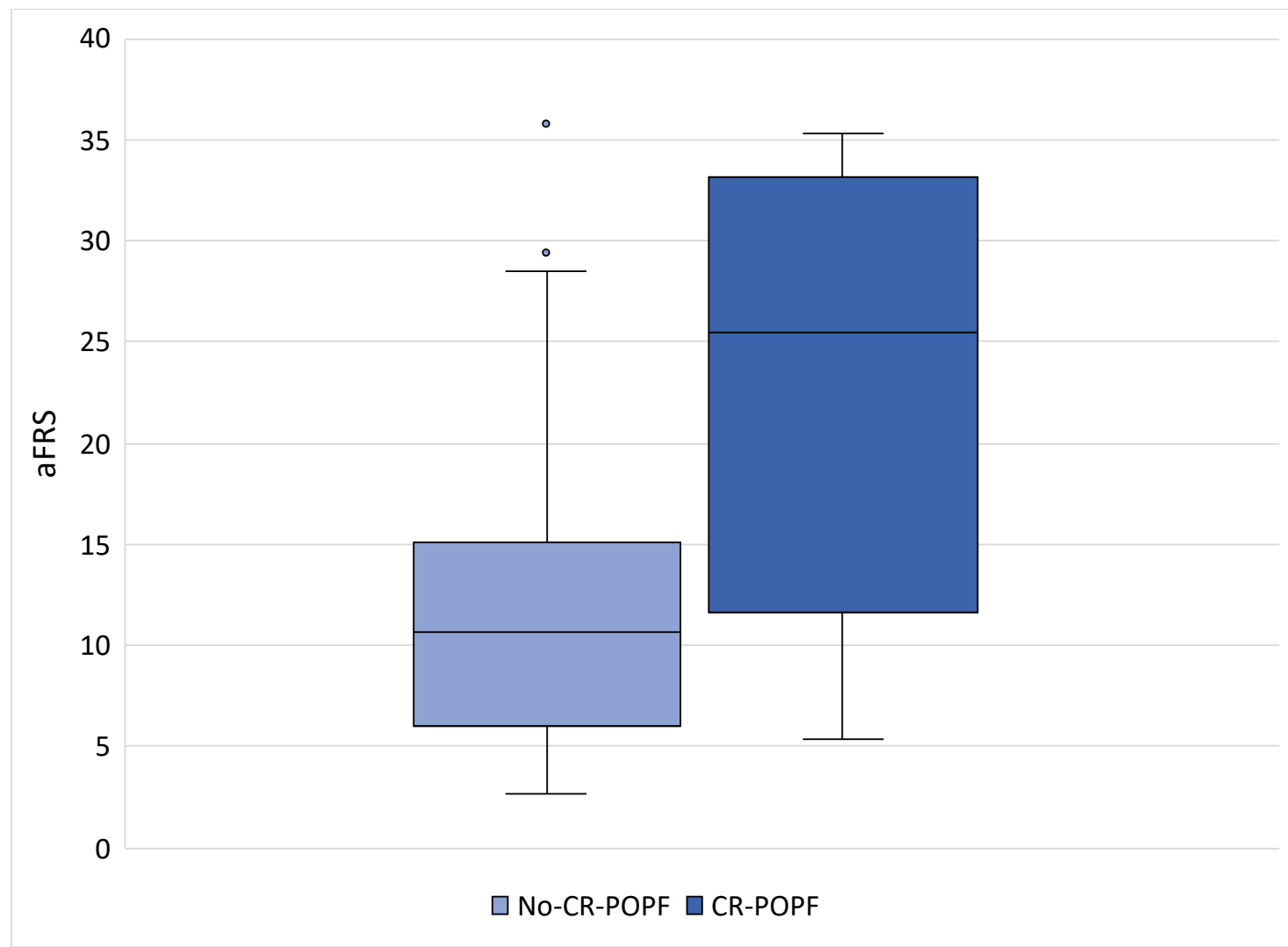

Fig. 3. Analysis of the distribution of aFRS values in relation to the occurrence of CR-POPF. The patients were divided into two groups: those with CR-POPF (on the right) and those without CR-POPF (on the left), represented by the two boxplots, with their corresponding aFRS values on the vertical axis. The aFRS values were not significantly different between patients of the two groups (Mann-Whitney U test: 59.5, $\mathrm{p}=0.12$ ). aFRS: alternative fistula risk score; CR-POPF: clinically relevant postoperative pancreatic fistula.

\section{Correlation between DFA and CR-POPF}

The mean DFA was 19,082 U/L and 2,388 U/L in the CR-POPF and non-CR-POPF groups, respectively. Considering DFA for risk stratification of the sample, 7 (17.5\%) patients presented values $\geq 5,000$ U/L, 5 (71.4\%) of whom evolved with CR-POPF. Contrarily, 33 (82.5\%) patients presented values $<5,000 \mathrm{U} / \mathrm{L}$, $1(3 \%)$ of whom evolved with CR-POPF. It was observed that the mean DFA of the 2 groups had very different magnitudes, indicating a higher discriminatory power of this variable.

The analysis of the variability of DFA in relation to the presence of CR-POPF demonstrated that the DFA medians differed significantly based on the presence or absence of CR-POPF (Fig. 4).

Both aFRS and DFA had higher median values in the CR-POPF compared to the non-CR-POPF group. However, unlike the previous analysis (aFRS and CR-POPF - Fig. 3), it was possible to completely separate the 
data in the boxplots in this analysis (DFA and CR-POPF - Fig. 4), which corroborates the strong discriminatory power of DFA.

The distribution of DFA values was non-parametric ( $\mathrm{p}<0.05$; Shapiro-Wilk normality test). There were differences between the CR-POPF and non-CR-POPF groups (Mann-Whitney U test: 27, p=0.004). This indicates that DFA has a greater discriminatory power for the occurrence of CR-POPF compared to aFRS.

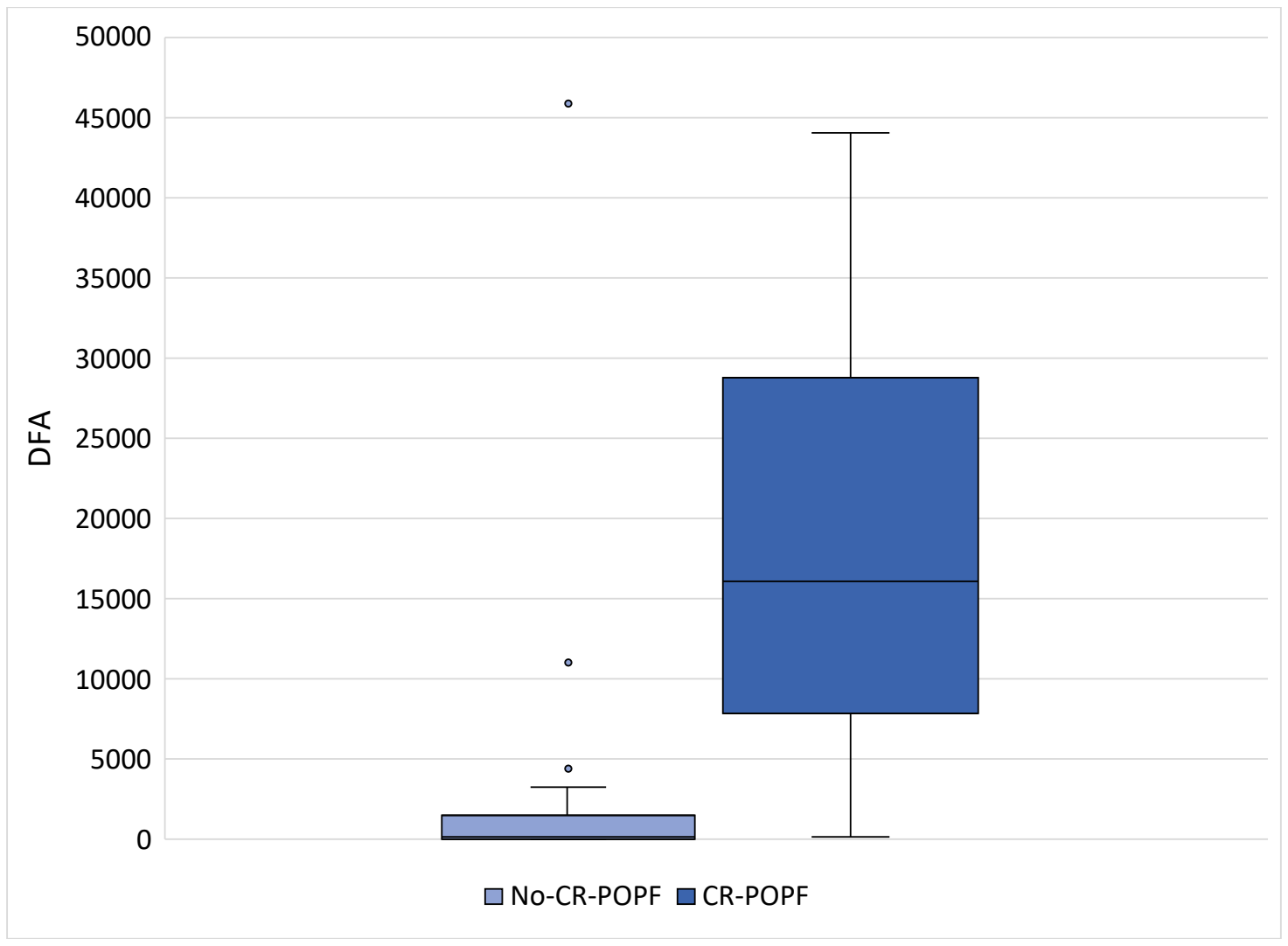

Fig. 4. Analysis of the distribution of DFA values in relation to the occurrence of CR-POPF. The patients were divided into two groups: those with CR-POPF (on the right) and those without CR-POPF (on the left), represented by the two boxplots, with their corresponding DFA values on the vertical axis. The DFA values were significantly different between the two groups (Mann-Whitney U test: $27, \mathrm{p}=0.004$ ).

DFA: first postoperative day drain fluid amylase; CR-POPF: clinically relevant postoperative pancreatic fistula.

\section{Comparing the predictive models}

The maximum accuracy of aFRS alone was 0.85 , with a value of $29.4 \%$. An Se of 0.33 and an Sp of $0.94(\mathrm{PPV}=0.50, \mathrm{NPV}=0.88)$ were observed for that cutoff. Using the cutoffs provided in the literature, for $\mathrm{aFRS}=5 \%$ and for $\mathrm{aFRS}=20 \%$, sensitivities were 1.00 and 0.66 , specificities were 0.17 and 0.85 , PPVs were 0.17 and 0.44 , NPVs were 1.00 and 0.93 and accuracies were 0.30 and 0.82 , respectively. 
The maximum accuracy of the DFA alone was 0.92 , with a value of 4,377 U/L. An Se of 0.83 and an Sp of $0.94(\mathrm{PPV}=0.71, \mathrm{NPV}=0.96)$ were observed for that cutoff. The metrics were similar for DFA=5,000 U/L. An 'accuracy freeze' occurred for DFA values above 4,377 U/L, in which the values of the confusion matrix coincided with the cutoff values obtained through the accuracy analysis and the cutoff above this value did not allow for the definition of new cutoff values.

For the combined model of aFRS $>20 \%+$ DFA $\geq 5,000 \mathrm{U} / \mathrm{L}$, the Se, Sp, PPV, NPV, and accuracy were $0.66,1.00,1.00,0.94$ and 0.95 , respectively.

The values of the metrics of the predictive models are summarized in Table 3.

Table 3. Metrics calculated for each predictive model, with the variables singly or combined: aFRS, DFA, and aFRS + DFA.

\begin{tabular}{lcccc}
\hline Metrics & aFRS $\leq \mathbf{5 \%}$ & aFRS $>\mathbf{2 0} \%$ & DFA $\geq \mathbf{5 , 0 0 0}$ U/L & aFRS $>\mathbf{2 0} \%+$ DFA $\geq \mathbf{5 , 0 0 0}$ U/L \\
\hline Se & 1.00 & 0.66 & 0.83 & 0.66 \\
\hline Sp & 0.17 & 0.85 & 0.94 & 1.00 \\
\hline PPV & 0.17 & 0.44 & 0.71 & 1.00 \\
\hline NPV & 1.00 & 0.93 & 0.96 & 0.94 \\
\hline Accuracy & 0.30 & 0.82 & 0.92 & 0.95
\end{tabular}

Se: sensitivity; Sp: specificity; PPV: positive predictive value; NPV: negative predictive value.

The ROC curve was plotted for the aFRS and DFA to analyze the different cutoff values and find the points of the greatest $\mathrm{Sp}$ and Se to differentiate the CR-POPF from the non-CR-POPF group (Fig. 5). The area under the curve (AUC) was obtained from the ROC curve for each predictive model: AUC (aFRS)=0.71; AUC $(\mathrm{DFA})=0.86$. 


\section{ROC curve}

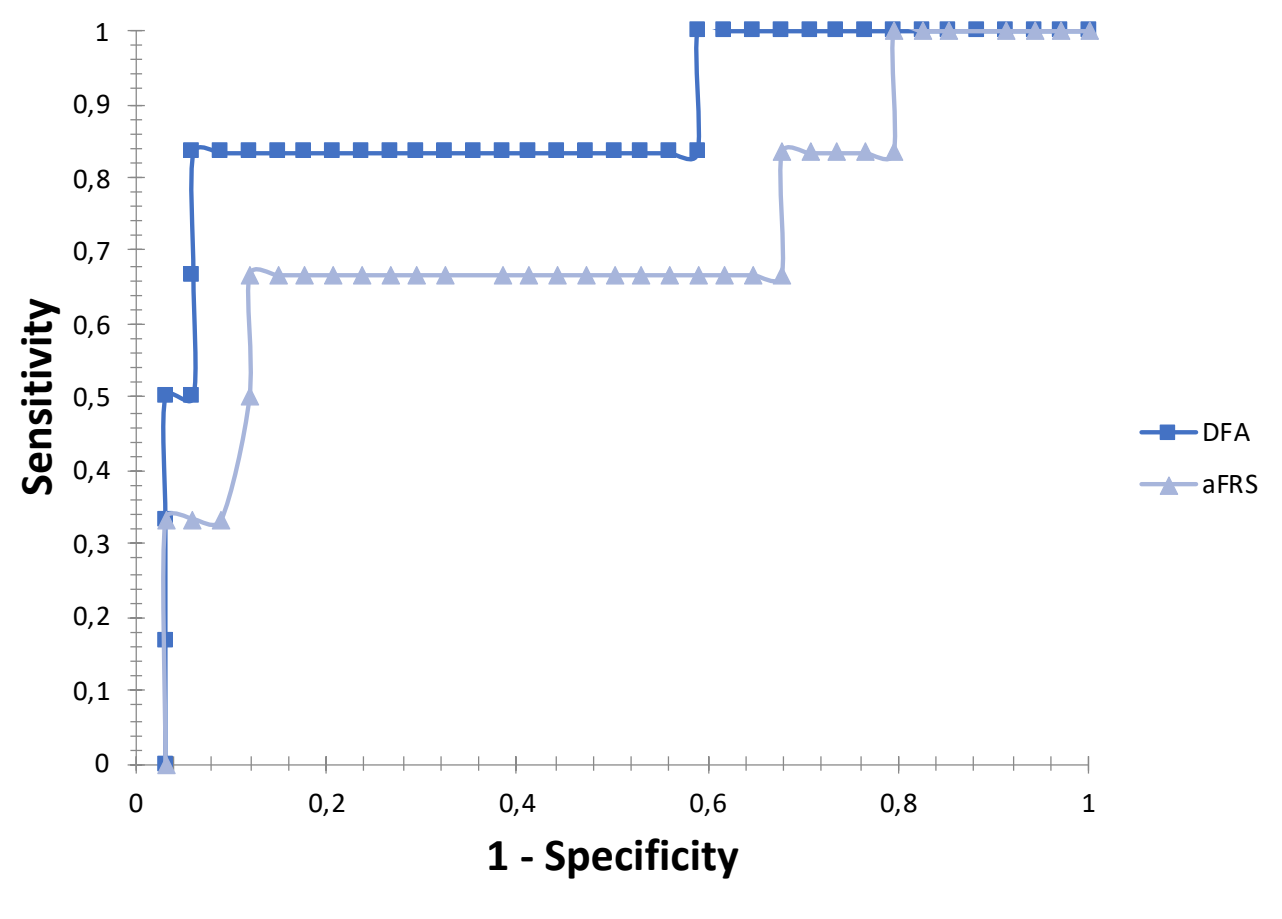

Fig. 5. ROC curve comparing the different cutoff values for aFRS and DFA and the corresponding AUC. AUC $($ aFRS $)=0.71 ;$ AUC $($ DFA $)=0.86$.

ROC: receiver operating characteristic; aFRS: alternative fistula risk score; DFA: first postoperative day drain fluid amylase; AUC: area under curve.

\section{Discussion}

CR-POPF is one of the most serious complications and is responsible for the high morbidity and mortality rates of PD. The morbidity rates range from 30 to $50 \%$ in large reference centers; however, the mortality rates have been reduced to less than $5 \%$ due to recent advances in perioperative management. ${ }^{1,2,12}$ The clinical impact of CR-POPF leads to significantly higher health costs by increasing hospital stay and the need for invasive interventions. ${ }^{3,4,12}$

This study assessed the association between aFRS and DFA and the occurrence of CR-POPF and compared the models for predicting the occurrence of CR-POPF.

The incidence of CR-POPF in the present study was 15\%, which is in agreement with that reported in the literature. ${ }^{1-4}$ The CR-POPF group showed a trend towards smaller Wirsung's duct diameters and greater BMIs, which are parameters that are used for calculating aFRS. ${ }^{4}$ 
The Wirsung's duct diameter and the pancreatic parenchyma texture are criteria considered subjective since these measurements are intraoperatively determined by the surgeon's autonomy. However, studies ${ }^{30,31}$ suggest that the determination of these criteria by experienced surgeons is accurate and intraoperative measurements can correlate with corresponding findings on preoperative images (three-phase computed tomography or nuclear magnetic resonance). ${ }^{32-34}$

Mungroop et al. ${ }^{4}$ have demonstrated that Se and Sp are improved by using the aFRS for predicting the occurrence of CR-POPF compared to the original FRS. Moreover, the aFRS has become more feasible in clinical practice as it does not use the histopathological examination report and the measurement of intraoperative bleeding, information that is required for calculating the original FRS. ${ }^{3}$ Furthermore, it is difficult for surgeons to calculate the original FRS since the measurement of intraoperative bleeding may not be accurate and the histopathological report is often only available in the late postoperative period. ${ }^{4}$

The calculation of the aFRS is inherent to the patient and its use in the literature has shown to be of great value for screening patients, especially those at low risk of CR-POPF occurrence. ${ }^{4-6,8,9}$ The lower values $($ aFRS $\leq 5 \%)$ in the present study, although not very accurate, have a high Se and NPV. As predicted by the low risk score (aFRS $\leq 5 \%$ ), none of the patients in the present study evolved with CR-POPF. Lao et al. ${ }^{5}$ retrospectively evaluated the external validation of aFRS in 370 patients who underwent PD and found a low accuracy (0.46) and high Se (0.92) and NPV (0.94). The present study corroborated these results and, thus, suggests the use of this indicator for selecting patients at low risk for CR-POPF.

The correlation of the aFRS alone with the CR-POPF showed a trend towards fistula occurrence since the aFRS value increased, which agrees with results found in the literature. ${ }^{4-6,8,9}$

DFA measurement is a simple procedure; however, it requires the presence of the abdominal drain. Bassi et al. ${ }^{12}$ correlated the DFA with the occurrence of CR-POPF and encouraged the strategy of early removal of the abdominal drain depending on DFA values. ${ }^{25}$ The cutoff provided in the literature ${ }^{11-24}$ ranged from 90 $\mathrm{U} / \mathrm{L}^{21}$ to $5,000 \mathrm{U} / \mathrm{L} .{ }^{12}$ Giglio et al. ${ }^{13}$ performed a meta-analysis with 13 studies and 4,416 patients to assess the accuracy of 11 different DFA cutoff values as predictors of CR-POPF, and found the highest Sp for the value of $5,000 \mathrm{U} / \mathrm{L}$.

The correlation between DFA and CR-POPF showed a trend towards fistula occurrence as DFA values increased, which is in agreement with findings from other studies. ${ }^{11-24}$ In the present case series, the DFA showed a high discriminatory power, since the means of the two groups had very different magnitudes. The DFA boxplot visually showed its strong predictive power by a significant independent separation of data. 
Moreover, the accuracy of the DFA for predicting the occurrence of CR-POPF was higher than that of the aFRS when the scores were analyzed individually. For these reasons, the DFA alone proved to be a strong predictive parameter for CR-POPF occurrence, and with a greater discriminatory power compared to the aFRS.

The most accurate model in the present study for predicting CR-POPF occurrence was aFRS $>20 \%+$ DFA $\geq 5,000 \mathrm{U} / \mathrm{L}$. There was an increase in accuracy when the scores were combined. Notably, this combination used the two values of $\mathrm{aFRS}^{4}$ and $\mathrm{DFA}^{12}$ obtained in the literature for determining the high risk for CR-POPF. The combination of these two scores is of great interest since their methods and advantages for predicting CRPOPF seem broad and overlapping. To our knowledge, this association between aFRS and DFA had not yet been studied.

Therefore, using the aFRS and DFA, it was possible to predict CR-POPF occurrence intraoperatively and on postoperative day 1 , respectively, with greater accuracy for the combined aFRS + DFA. Although our sample is small, this information may help in further studies to identify patients at high risk for CR-POPF (aFRS $>20 \%$ and DFA $\geq 5,000 \mathrm{U} / \mathrm{L}$ ) who may be candidates for more rigorous postoperative monitoring, and patients at low risk for CR-POPF (aFRS $\leq 20 \%$ and DFA $<5,000 \mathrm{U} / \mathrm{L}$ ) who may be candidates for fast-track protocols such as early return to normal diet, removal of the abdominal drain, and discharge. Nevertheless, our results need to be confirmed with prospective clinical studies. ${ }^{23-29,35-43}$

Kawai et al. ${ }^{29}$ argued that early discharge (around postoperative day 5) can be safe and economically beneficial for patients with good progression following PD, while others encourage the omission of the prophylactic drain in patients with low risk of CR-POPF, as well as its early removal when the DFA is $<5,000$ U/L. ${ }^{23-25,27-29,35-39,41-43}$ However, most institutions still use the abdominal drain routinely and remove it on or after postoperative day $7 .^{29}$

Postoperative management is planned based on existing complications, which affect the length of hospital stay and the patient's morbidity and mortality. ${ }^{12}$ Regarding the length of hospital stay and duration of abdominal drain use, the present case series showed a trend for the CR-POPF group to stay longer in the hospital compared to the non-CR-POPF group, and an association between the decision to remove the drain and the decision to discharge.

This study was limited by the small sample size. Moreover, it was a retrospective and observational cohort study. The association between aFRS and DFA may help differ the patients with low risk from those with high risk of developing CR-POPF, but further studies with a larger number of patients are required to confirm the possible prediction of CR-POPF occurrence with the combined use of the scores. 


\section{Conclusion}

The data obtained in this study demonstrated that the combined use of aFRS and DFA increased the accuracy for predicting CR-POPF in patients who underwent PD. Prospective studies with a larger number of cases are required to confirm these results.

\section{Acknowledgements}

Source of funding: None.

Disclosure statement: Declarations of Interest and Competing Interestst: NONE.

\section{References}

1. Bassi, C. et al. Postoperative pancreatic fistula: an international study group (ISGPF) definition. Surgery. 2005 Jul;138(1):8-13.

2. Bassi, C. et al. The 2016 update of the International Study Group (ISGPS) definition and grading of postoperative pancreatic fistula: 11 Years After. Surgery. 2017 Mar;161(3):584-591.

3. Callery, M.P., Pratt, W.B., Kent, T.S., Chaikof, E.L. \& Vollmer, C.M. A prospectively validated clinical risk score accurately predicts pancreatic fistula after pancreatoduodenectomy. J Am Coll Surg. 2013 Jan;216(1):114.

4. Mungroop, T.H. et al. Alternative Fistula Risk Score for Pancreatoduodenectomy (a-FRS): Design and International External Validation. Ann Surg. 2019 May;269(5):937-943.

5. Lao, M. et al. External validation of alternative fistula risk score (a-FRS) for predicting pancreatic fistula after pancreatoduodenectomy. HPB (Oxford). 2020 Jan;22(1):58-66.

6. Ryu, Y. et al. Validation of original and alternative fistula risk scores in postoperative pancreatic fistula. $J$ Hepatobiliary Pancreat Sci. 2019 Aug;26(8):354-359.

7. Mungroop, T.H. et al. Updated Alternative Fistula Risk Score (ua-FRS) to Include Minimally Invasive Pancreatoduodenectomy: Pan-European Validation. Ann Surg. 2021 Feb 1;273(2):334-340.

8. Shinde, R.S. et al. External validation and comparison of the original, alternative and updated-alternative fistula risk scores for the prediction of postoperative pancreatic fistula after pancreatoduodenectomy. Pancreatology. 2020 Jun;20(4):751-756. 
9. Hayashi, H. et al. Comparison of three fistula risk scores after pancreatoduodenectomy: A single-institution retrospective study. Asian J Surg. 2021 Jan;44(1):143-146.

10. Yamaguchi, M. et al. Prediction of pancreatic fistula by amylase levels of drainage fluid on the first day after pancreatectomy. Hepatogastroenterology. 2003 Jul-Aug;50(52):1155-8.

11. Shinchi, H., Wada, K. \& Traverso, L.W. The usefulness of drain data to identify a clinically relevant pancreatic anastomotic leak after pancreaticoduodenectomy? J Gastrointest Surg. 2006 Apr;10(4):490-8.

12. Molinari, E. et al. Amylase value in drains after pancreatic resection as predictive factor of postoperative pancreatic fistula: results of a prospective study in 137 patients. Ann Surg. 2007 Aug;246(2):281-7.

13. Giglio, M.C. et al. Meta-analysis of drain amylase content on postoperative day 1 as a predictor of pancreatic fistula following pancreatic resection. Br J Surg. 2016 Mar;103(4):328-36.

14. Davidson, T.B., Yaghoobi, M., Davidson, B.R. \& Gurusamy, K.S. Amylase in drain fluid for the diagnosis of pancreatic leak in post-pancreatic resection. Cochrane Database Syst Rev. 2017 Apr 7;4(4):CD012009.

15. Yang, J., Huang, Q. \& Wang, C. Postoperative drain amylase predicts pancreatic fistula in pancreatic surgery: A systematic review and meta-analysis. Int J Surg. 2015 Oct;22:38-45.

16. Liu, Y., Li, Y., Wang, L. \& Peng, C.J. Predictive value of drain pancreatic amylase concentration for postoperative pancreatic fistula on postoperative day 1 after pancreatic resection: An updated meta-analysis. Medicine (Baltimore). 2018 Sep;97(38):e12487.

17. Lu, X. et al. Systematic Review and Meta-Analysis of Pancreatic Amylase Value on Postoperative Day 1 After Pancreatic Resection to Predict Postoperative Pancreatic Fistula. Medicine (Baltimore). 2016 Feb;95(5):e2569.

18. Conlon, K.C. et al. Prospective randomized clinical trial of the value of intraperitoneal drainage after pancreatic resection. Ann Surg. 2001 Oct;234(4):487-93.

19. Maggino, L. et al. Identification of an Optimal Cut-off for Drain Fluid Amylase on Postoperative Day 1 for Predicting Clinically Relevant Fistula After Distal Pancreatectomy: A Multi-institutional Analysis and External Validation. Ann Surg. 2019 Feb;269(2):337-343.

20. Dugalic, V.D. et al. Drain amylase value as an early predictor of pancreatic fistula after cephalic duodenopancreatectomy. World J Gastroenterol. 2014 Jul 14;20(26):8691-9.

21. Lee, C.W. et al. Low drain fluid amylase predicts absence of pancreatic fistula following pancreatectomy. $J$ Gastrointest Surg. 2014 Nov;18(11):1902-10. 
22. Amico, E.C., Azevedo, Í.M., Fernandes, M.V.L., Reis, M.A. \& João, S.A. Drain amylase on the first postoperative day of Whipple surgery: what value is the best predictor for early drain removal? Arq Bras Cir Dig. 2018 Mar 1;31(1):e1345.

23. Newhook, T.E. et al. Early postoperative drain fluid amylase in risk-stratified patients promotes tailored postpancreatectomy drain management and potential for accelerated discharge. Surgery. 2020 Feb;167(2):442-447.

24. Villafane-Ferriol, N. et al. Sequential drain amylase to guide drain removal following pancreatectomy. $H P B$ (Oxford). 2018 Jun;20(6):514-520.

25. Bassi, C. et al. Early versus late drain removal after standard pancreatic resections: results of a prospective randomized trial. Ann Surg. 2010 Aug;252(2):207-14.

26. Maggino, L. et al. Decoding Grade B Pancreatic Fistula: A Clinical and Economical Analysis and Subclassification Proposal. Ann Surg. 2019 Jun;269(6):1146-1153.

27. Bertens, K.A. et al. What is a better predictor of clinically relevant postoperative pancreatic fistula (CR-POPF) following pancreaticoduodenectomy (PD): postoperative day one drain amylase (POD1DA) or the fistula risk score (FRS)? HPB (Oxford). 2017 Jan;19(1):75-81.

28. Villafane-Ferriol, N. et al. Evidence Versus Practice in Early Drain Removal After Pancreatectomy. J Surg Res. 2019 Apr;236:332-339.

29. Kawai, M. et al. Early removal of prophylactic drains reduces the risk of intra-abdominal infections in patients with pancreatic head resection: prospective study for 104 consecutive patients. Ann Surg. 2006 Jul;244(1):1-7.

30. Belyaev, O. et al. Assessment of pancreatic hardness - surgeon versus durometer. J Surg Res. 2010 Jan;158(1):53-60.

31. Hong, T.H. et al. Pancreatic hardness: Correlation of surgeon's palpation, durometer measurement and preoperative magnetic resonance imaging features. World J Gastroenterol. 2017 Mar 21;23(11):2044-2051.

32. Lee, S.E. et al. Measurement of pancreatic fat by magnetic resonance imaging: predicting the occurrence of pancreatic fistula after pancreatoduodenectomy. Ann Surg. 2010 May;251(5):932-6.

33. Hashimoto, Y. et al. Dual-phase computed tomography for assessment of pancreatic fibrosis and anastomotic failure risk following pancreatoduodenectomy. J Gastrointest Surg. 2011 Dec;15(12):2193-204.

34. Kantor, O. et al. Using the NSQIP Pancreatic Demonstration Project to Derive a Modified Fistula Risk Score for Preoperative Risk Stratification in Patients Undergoing Pancreaticoduodenectomy. J Am Coll Surg. 2017 May;224(5):816-825. 
35. Villafane-Ferriol, N. et al. Evidence-Based Management of Drains Following Pancreatic Resection: A Systematic Review. Pancreas. 2018 Jan;47(1):12-17.

36. Sutcliffe, R.P. et al. Implementation of an Enhanced Recovery Pathway After Pancreaticoduodenectomy in Patients with Low Drain Fluid Amylase. World J Surg. 2015 Aug;39(8):2023-30.

37. McMillan, M.T. et al. The value of drains as a fistula mitigation strategy for pancreatoduodenectomy: something for everyone? Results of a randomized prospective multi-institutional study. J Gastrointest Surg. 2015 Jan;19(1):21-30; discussion 30-1.

38. Van Buren, G. et al. A randomized prospective multicenter trial of pancreaticoduodenectomy with and without routine intraperitoneal drainage. Ann Surg. 2014 Apr;259(4):605-12.

39. Heslin, M.J. et al. Is intra-abdominal drainage necessary after pancreaticoduodenectomy? J Gastrointest Surg. 1998 Jul-Aug;2(4):373-8.

40. Jeekel, J. et al. No abdominal drainage after Whipple's procedure. Br J Surg. 1992 Feb;79(2):182.

41. Correa-Gallego, C. et al. Operative drainage following pancreatic resection: analysis of 1122 patients resected over 5 years at a single institution. Ann Surg. 2013 Dec;258(6):1051-8.

42. McMillan, M.T. et al. Drain Management after Pancreatoduodenectomy: Reappraisal of a Prospective Randomized Trial Using Risk Stratification. J Am Coll Surg. 2015 Oct;221(4):798-809.

43. McMillan, M.T. et al. Multicenter, Prospective Trial of Selective Drain Management for Pancreatoduodenectomy Using Risk Stratification. Ann Surg. 2017 Jun;265(6):1209-1218.

\section{Author Contributions Statement:}

Karina Scalabrin Longo ${ }^{1}$, Thiago Bassaneze ${ }^{2}$, Rogério Tadeu Palma ${ }^{3}$, Jaques Waisberg ${ }^{4}$

(1) Conception and design; data collect; analysis and interpretation of data; drafting and writing the article.

(2) Conception and design; revising the article critically for important intellectual content.

(3) Revising the article critically for important intellectual content.

(4) Revising the article critically for important intellectual content.

The authors declare that they have no known competing financial interests or personal relationships that could have appeared to influence the work reported in this paper.

\section{Figure Legends}

Fig. 1. Selection of the studied patients and occurrence or not of clinically relevant postoperative pancreatic fistula. CR-POPF: clinically relevant postoperative pancreatic fistula; BL: biochemical leak; POPF: 
postoperative pancreatic fistula, Grade B POPF: fistula requiring a change in postoperative management. Grade C POPF: grade B with organ failure, clinical instability, need for reoperation, or death.

Fig. 2. Analysis of the distribution of aFRS categories in relation to the occurrence of CR-POPF. The patients were divided into three columns, according to aFRS categories: those with low- (aFRS 0\% to 5\%), those with intermediate- (aFRS >5\% to 20\%), and those with high-risk (aFRS >20\%) for developing CR-POPF. Each column was divided into patients who developed CR-POPF (dark blue) and patients who not developed CR-POPF (light blue). aFRS: alternative fistula risk score; CR-POPF: clinically relevant postoperative pancreatic fistula.

Fig. 3. Analysis of the distribution of aFRS values in relation to the occurrence of CR-POPF. The patients were divided into two groups: those with CR-POPF (on the right) and those without CR-POPF (on the left), represented by the two boxplots, with their corresponding aFRS values on the vertical axis. The aFRS values were not significantly different between patients of the two groups (Mann-Whitney $U$ test: 59.5 , $\mathrm{p}=0.12$ ). aFRS: alternative fistula risk score; CR-POPF: clinically relevant postoperative pancreatic fistula.

Fig. 4. Analysis of the distribution of DFA values in relation to the occurrence of CR-POPF. The patients were divided into two groups: those with CR-POPF (on the right) and those without CR-POPF (on the left), represented by the two boxplots, with their corresponding DFA values on the vertical axis. The DFA values were significantly different between the two groups (Mann-Whitney $U$ test: 27, $\mathrm{p}=0.004$ ). DFA: first postoperative day drain fluid amylase; CR-POPF: clinically relevant postoperative pancreatic fistula.

Fig. 5. ROC curve comparing the different cutoff values for aFRS and DFA and the corresponding AUC. AUC $($ aFRS $)=0.71$; AUC $($ DFA $)=0.86$. ROC: receiver operating characteristic; aFRS: alternative fistula risk score; DFA: first postoperative day drain fluid amylase; AUC: area under curve. 